\title{
GRAVITATIONAL VIRIALIZATION OF MOLECULAR CLOUD FRAGMENTS
}

\author{
Anthony WHITWORTH \\ Department of Physics, \\ University of Wales College of Cardiff, \\ PO Box 913, Cardiff CF1 $3 T H$, \\ Wales, UK.
}

\begin{abstract}
We argue that if gravitational interactions play an important role in the amplification and transfer of turbulent energy between sub-clouds of different scales in a GMC, then the Second Law of Thermodynamics requires that the transfer occur from smaller to larger scales, and hence that the seed-energy be injected on relatively small scales.
\end{abstract}

\section{Introduction}

Scalo and Pumphrey (1982) have proposed that when an interstellar cloud contracts and fragments into sub-clouds, collisions between the sub-clouds are on average not very dissipative. A large fraction of the gravitational energy released by the contracting parent-cloud is channelled initially into randomized bulk kinetic energy of the sub-clouds. The parent-cloud is effectively virialized, ir: the sense that its subsequent contraction proceeds quasistatically on a time-scale which is much longer than a freefall time.

Scalo (1987) has termed this process gravitational virialization. He suggests that it may play an important role in the evolution of GMCs, as a means of generating turbulent kinetic energy and transferring this energy from one scale to another.

\section{Assumptions}

We assume - but do not unequivocally believe - (i) that Larson's relations $(R \propto$ $\left.M^{\alpha}, \alpha \sim 0.5 ; \Delta v \propto M^{\beta}, \beta \sim 0.25\right)$ are real, rather than just an observational selection effect; (ii) that all clouds and sub-clouds subscribe to these relations; (iii) that all clouds and sub-clouds are virialized $(\alpha=1-2 \beta)$; (iv) that all clouds and 
sub-clouds belong to an homologous sequence; and (v) that there is an evolutionary connection between clouds and sub-clouds of different scales.

\section{The Second Law of Thermodynamics}

The 2nd Law of Thermodynamics requires evolution to proceed in that sense in which heat (here in the form of cooling radiation) flows out of the system (which is effectively at a temperature 10-100K) into the surroundings (which are effectively at a temperature 3-10K). Hence there must be a net release of gravitational potential energy, with half of the gravitational energy released going towards maintaining virialization (i.e. feeding the turbulent velocity dispersion), and the other half escaping as radiation.

\section{Up $\rightarrow$ Down Gravitational Virialization}

Consider a static, cold, spherical parent-cloud of mass $M$ and radius $R_{o}$, which contracts and breaks up into $N$ sub-clouds, each of mass $m=M / N$. If a fraction $F$ of the gravitational potential energy released by the contracting parent-cloud is channelled into random bulk motions of the sub-clouds, whilst the rest is radiated away, the final virialized radius of the parent-cloud is

$$
R=R_{o} \frac{(2 F-1)}{2 F}
$$

Gravitational virialization therefore requires $F>1 / 2$.

Now consider the sub-clouds. Any gravitational potential energy they release in contracting to a radius $r_{o}=R N^{-1 / 3}$ has been used in virializing the parent-cloud. Consequently, if the sub-clouds are to be gravitationally virialized in their turn, they must contract still further to a radius

$$
r=r_{o} \frac{(2 f-1)}{2 f}=R N^{-1 / 3} \frac{(2 f-1)}{2 f}
$$

Here $f$ is the fraction of the self gravitational potential energy of a sub-cloud which is channelled into random bulk motions of the sub-sub-clouds. Again gravitational virialization requires $f>1 / 2$.

Larson's relations give $r=R N^{-\alpha}$, so equation (2) reduces to

$$
N=\left\{\frac{2 f}{(2 f-1)}\right\}^{3 /(3 \alpha-1)}
$$

The following table gives values of $N$ for representative combinations of $f$ and $\alpha$. 


\begin{tabular}{|l|l|l|l|l|}
\hline$N$ & $f=0.9$ & $f=0.8$ & $f=0.7$ & $f=0.6$ \\
\hline$\alpha=0.4$ & $1.9 \times 10^{5}$ & $2.5 \times 10^{6}$ & $1.4 \times 10^{8}$ & $4.7 \times 10^{11}$ \\
\hline$\alpha=0.5$ & 130 & 362 & 1838 & 46656 \\
\hline$\alpha=0.6$ & 21 & 40 & 110 & 828 \\
\hline
\end{tabular}

We conclude that $N$ has to be very large (unless $f$ is very high, i.e. gravitational virialization is nearly $100 \%$ efficient, and $\alpha$ is very much larger than inferred from observation). The parent-cloud must shatter into a myriad of much smaller sub-clouds. This is in stark contrast to the conventional picture of hierarchical fragmentation (e.g. Zinnecker 1984) in which at each level of the hierarchy a parentcloud breaks into just a few sub-clouds, and the process then repeats itself in a more-or-less self-similar manner over a large spatial range.

\section{Down $\rightarrow$ Up Gravitational Virialization}

The alternative to the scenario outlined above is obtained if one turns hierarchical fragmentation on its head. Hierarchical fragmentation is in any case seriously flawed (e.g. Whitworth 1980), and without observational basis. If we turn it on its head, we have a scenario in which the dominant injection of turbulent energy is on small scales, and this energy is then transferred to larger scales and simultaneously amplified by gravitational virialization.

To establish that this will work, we consider an ensemble of $N$ independent, virialized sub-clouds, each of mass $m$ and radius $r$. If these sub-clouds combine to form a single collective-cloud with mass $M=N m$ and radius $R=r N^{\alpha}$, the net gravitational energy released is positive (and hence the process is permitted by the 2nd Law of Thermodynamics) provided

$$
N^{(1-\alpha)}>1-N^{(1-3 \alpha) / 3}
$$

This condition is satisfied for any positive integral $N$ as long as $\alpha<1$. In other words, the gravitational potential energy released by the sub-clouds falling together is more than enough to puff them up individually to the lower density required for the collective-cloud by Larson's relations.

\section{Conclusions}

We conclude that gravitational virialization is only likely to work if energy is transferred in the opposite sense to Kolmogorov turbulence, i.e. down $\rightarrow$ up, from smaller scales to larger ones. Although this goes against the theoretical concept of hierarchical fragmentation, there does not appear to be any unequivocal observational 
evidence to rule out the possibility that this is what occurs in GMCs. Other arguments supporting the down $\rightarrow$ up transfer and amplification of turbulent energy in GMCs will be assembled in a future paper.

\section{References}

Scalo, J.M., 1987, in Interstellar Processes

(eds. D.J. Hollenbach, H.A. Thronson, Jnr.; Reidel, Dordrecht), 349

Scalo, J.M., Pumphrey, W.A., 1982, Astrophys. J. 258, L29

Whitworth, A.P., 1980, in Giant Molecular Clouds in the Galaxy

(eds. P.M. Solomon, M.G. Edmunds; Pergamon, Oxford), 285

Zinnecker, H., 1984, Mon. Not. roy. astron. Soc. 210, 43 